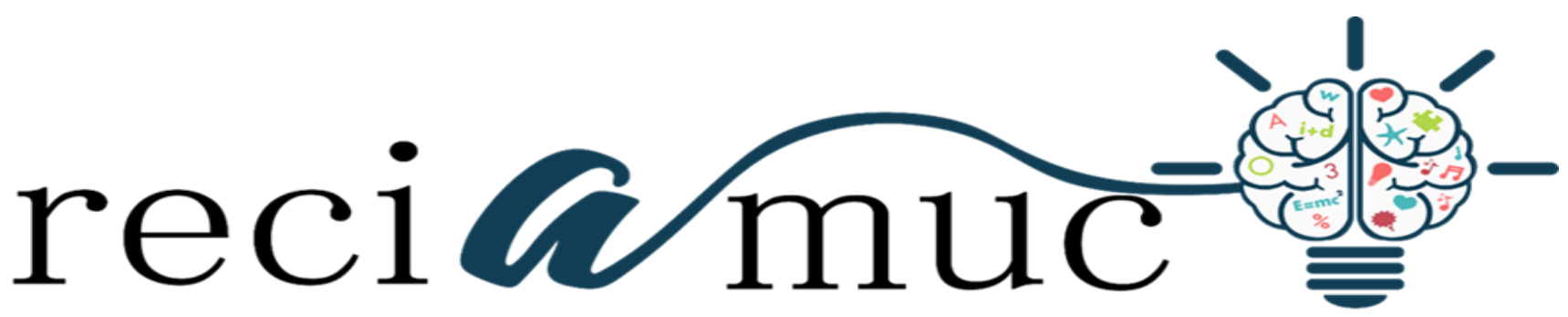

Revista cientifica de investigación actualización del mundo de las ciencias

Nathalie María Barriga Reyes a ; Hypatia Amariles Ganan Macías b; Renato Paolo
Patiño Andrade ${ }^{\text {c; }}$ María Monserrate Ganchozo Pincay ${ }^{\text {d }}$

Falla en la cicatrización post operatoria en apendicitis

Failure in post operative cicatrization in appendicitis

Revista Científica de Investigación actualización del mundo de las Ciencias. Vol. 3 núm., 3, julio, ISSN: 2588-0748, 2018, pp. 789-816

DOI: $10.26820 /$ reciamuc/3.(3).julio.2019.789-916

URL: $\underline{\text { htp: }: / / \text { reciamuc.com/index.php/RECIAMUC/article/view/304 }}$

Código UNESCO: 3213.01 Cirugía Abdominal

Tipo de Investigación: Artículo de Revisión

() RECIAMUC; Editorial Saberes del Conocimiento, 2019

Recibido: 28/04/2019

Aceptado: 19/05/2019

Publicado: 01/07/2019

Correspondencia: nathyb_24@hotmail.com
a. Médico; Investigador Independiente; Guayaquil, Ecuador; nathyb_24@ hotmail.com
b. Médico; Investigador Independiente; Guayaquil, Ecuador; patitaganan@yahoo.es
c. Médico; Investigador Independiente; Guayaquil, Ecuador; po_rp69@ hotmail.com
d. Médico; Investigador Independiente; Guayaquil, Ecuador; gp mayte@ hotmail.com 


\section{Falla en la cicatrización post operatoria en apendicitis}

Vol. 3, núm. 3., (2019)

Nathalie María Barriga Reyes; Hypatia Amariles Ganan Macías; Renato Paolo Patiño Andrade; María Monserrate Ganchozo Pincay

\section{RESUMEN}

La apendicectomía, representa la cirugía de urgencia más común en los servicios quirúrgicos del mundo entero, esta patología no está exenta de complicaciones a pesar de que los avances tecnológicos de cirugía, de anestesiología y reanimación, reducen al mínimo el traumatismo operatorio. Su complicación más común es la del sitio operatorio (o falla de cicatrización), a la misma le siguen los abscesos intra abdominales y de las gastrointestinales (íleo paralítico posoperatorio). Las causas que la condicionan, son aún poco conocidas, por lo que su determinación sería importante para disminuir la morbilidad y mortalidad post operatorias. Esta afección médica de orden post operatorio inicia cuando ingresan bacterias en el sitio de la cirugía. Las infecciones pueden retardar el proceso de cicatrización y pueden extenderse a tejidos u órganos cercanos, o a zonas distantes a través del torrente circulatorio. El tratamiento de las heridas infectadas puede incluir lo siguiente: Antibióticos, Drenaje de los abscesos (acumulaciones de pus bajo la piel que se producen por la infección). Apertura de la incisión para eliminar el material infectado. Es importante resaltar que, la infección del sitio operatorio depende de muchos factores, entre los que se citan: la virulencia del germen causal, la respuesta del paciente a la infección, la habilidad del cirujano, el criterio para determinar que existe una infección, así como el estado del apéndice en el acto quirúrgico, señalando que su frecuencia es menor para las apendicitis tempranas que los de las perforadas. Los pacientes que se intervienen con un estado físico más precario tienen peor pronóstico, porque aumentan las posibilidades de presentar complicaciones post operatorias. La frecuencia y gravedad de esta afección todavía cobra vidas humanas de modo innecesario y constituye un tema de actualidad por su repercusión económica, social y científica.

Palabras Claves: Apendicitis Aguda; Cirugía; Complicaciones Médicas; Factores de Riesgo; Abscesos. 


\title{
Falla en la cicatrización post operatoria en apendicitis
}

Vol. 3, núm. 3., (2019)

Nathalie María Barriga Reyes; Hypatia Amariles Ganan Macías; Renato Paolo Patiño Andrade; María Monserrate Ganchozo Pincay

\begin{abstract}
Appendectomy, represents the most common emergency surgery in surgical services worldwide, this pathology is not without complications despite technological advances in surgery, anesthesiology and resuscitation, minimizing operative trauma. Its most common complication is that of the operative site (or failure to heal), followed by intra-abdominal abscesses and gastrointestinal abscesses (postoperative paralytic ileus). The causes that condition it are still poorly understood, so its determination would be important to reduce post-operative morbidity and mortality. This post-operative medical condition begins when bacteria enter the surgical site. Infections can slow the healing process and can spread to nearby tissues or organs, or to distant areas through the bloodstream. Treatment of infected wounds may include the following: Antibiotics, Abscess drainage (accumulations of pus under the skin that are produced by the infection). Opening the incision to remove the infected material. It is important to emphasize that infection of the operative site depends on many factors, among which are cited: the virulence of the causal germ, the patient's response to the infection, the skill of the surgeon, the criteria to determine that an infection exists, as well as the status of the appendix in the surgical act, noting that its frequency is lower for early appendicitis than the perforated ones. Patients who intervene with a more precarious physical condition have a worse prognosis, because they increase the possibilities of presenting postoperative complications. The frequency and severity of this condition still take human lives unnecessarily and is a topical issue due to its economic, social and scientific impact.
\end{abstract}

Key Words: Acute Appendicitis; Surgery; Medical Complications; Risk Factors; Abscesses. 


\section{Falla en la cicatrización post operatoria en apendicitis}

Vol. 3, núm. 3., (2019)

Nathalie María Barriga Reyes; Hypatia Amariles Ganan Macías; Renato Paolo Patiño Andrade; María Monserrate Ganchozo Pincay

\section{Introducción.}

La apendicitis aguda, representa en el mundo entero una de las patologías de mayor incidencia en los centros Hospitalarios, lo que hace que esta enfermedad represente un problema de salud relevante, tanto por la alta frecuencia en la población general, como por el consumo de recursos sanitarios hospitalarios que su tratamiento y complicaciones genera, lo que hace que la misma sea entendida por el sector salud como un problema de salud pública. Representa una anomalía cuya ocurrencia va casi paralela al desarrollo del tejido linfoide del individuo, con una prevalencia máxima en el adulto joven, lo que lleva a que siga siendo una intervención quirúrgica temprana. (Gumter, 2016).

En virtud de este planteamiento se destaca que, países latinoamericanos comprenden una alta incidencia de pacientes con esta enfermedad, la cual representa una de las cinco causas más comunes de litigios basados en malas prácticas ocurridas en estas Naciones, teniendo como base las siguientes causas: mala praxis médica desarrollada por algunos cirujanos, siendo las patologías que más destacan las referidas a los fallos en las historias clínicas y diagnóstico previo de la enfermedad, la demora en el tratamiento, la demora y diagnóstico equívoco, automedicación, falla en la cicatrización, manejo inadecuado en la atención primaria y en la realización del tratamiento definitivo; factores éstos que en su efecto incrementan la morbilidad y mortalidad relacionada con esta causa.

Tales afirmaciones llevan a establecer que el diagnóstico de apendicitis aguda es primariamente clínico, siendo su principal manifestación el dolor abdominal, el cual se inicia en epigastrio, es de intensidad moderada y en el transcurso del tiempo se localiza en el cuadrante 


\section{Falla en la cicatrización post operatoria en apendicitis}

Vol. 3, núm. 3., (2019)

Nathalie María Barriga Reyes; Hypatia Amariles Ganan Macías; Renato Paolo Patiño Andrade; María Monserrate Ganchozo Pincay

inferior derecho, sus variaciones son cambiantes y dependen en mayor grado, de la situación anatómica del apéndice, la cual refiere y contribuye de gran forma con la identificación de la fase somática del dolor, la cual en muchas ocasiones viene acompañada de anorexia, náuseas y vómitos o constipación

En correspondencia a los planteamientos dados, se refiere lo descrito por la Organización Mundial de la Salud, (Salud, 2017), la cual, a través de datos epidemiológicos refiere::

En Chile para el año 2016 se pudo observar una morbilidad del 75\% de casos de pacientes operados de apendicitis agudas, demostrándose con ello que esta patología tiene una prevalencia que va entre el 8 al 12\% de la población. Por su parte, en Perú estadísticas emanadas de la Organización Mundial de la Salud OMS demostraron que las cirugías por apéndice arrojaron un 35\% de casos con complicaciones generadas en el sitio operatorio; pacientes éstos que requirieron de hospitalización; convirtiéndose de esta manera en un problema epidemiológico. En el 2017, la frecuencia de apendicitis no complicada en estas Naciones, fue de alrededor de 33,25 por cada 100.000 habitantes; mientras que, en el 2017 fue de 35,32 por cada 100.000 habitantes y en el 2018 fue de 34,87 por cada 100.000 habitantes. (p.23).

En unión de ello, el Centro para el Control y Prevención de Enfermedades (CDS, 2017), señala se pudo identificar la presencia de 400.000 casos de pacientes con cirugía de apendicitis, en condiciones post operatorias que presentan complicaciones, destacándose entre ellas, la peritonitis y las dadas por fallas de cicatrización reconocidas como infecciones del sitio operatorio (ISO); esta última de gran incidencia a nivel mundial, identificada de forma anual en 


\section{Falla en la cicatrización post operatoria en apendicitis}

Vol. 3, núm. 3., (2019)

Nathalie María Barriga Reyes; Hypatia Amariles Ganan Macías; Renato Paolo Patiño Andrade; María Monserrate Ganchozo Pincay

los hospitales de Estados Unidos, dando lugar a varios miles de millones de dólares en costos médicos directos que son potencialmente evitables. (p.17).

Corresponde señalar que, la infección del sitio operatorio (ISO), continúa siendo una de las complicaciones más frecuentes de los pacientes sometidos a procedimientos quirúrgicos. Pese a los importantes avances en el conocimiento médico acerca de la fisiología de la cicatrización y los factores de riesgo de infección y de los inobjetables progresos en la tecnología científica de la asepsia y la antisepsia, la infección del sitio operatorio está entre las dos primeras causas de infección nosocomial en el mundo. (Humt, 2017).

Según lo expuesto, la apendicitis representa una patología muy común, esta afección aguda se caracteriza por ser muy propensa a complicaciones, siendo el riesgo de infección del sitio operatorio muy frecuente y depende del procedimiento quirúrgico realizado durante la cirugía y de la presencia de factores de riesgo. De allí, que el conocimiento de dichos factores de riesgo es muy importante, por cuanto permiten estratificar adecuadamente las diferentes intervenciones que son realizadas, y así, controlar las infecciones de una forma más racional.

Existen factores de riesgos que se asocian a la infección de sitio operatorio post apendicetomía convencional; conocer dichos factores permitirá al médico cirujano tomar medidas de prevención frente al problema, lo cual aportará dos beneficios; primero, garantizar una óptima recuperación del paciente, mejorar el servicio de atención y reducir la frecuencia de infecciones intrahospitalarias; segundo, reducir el tiempo de hospitalización de la persona, lo que conlleva a disminuir el consumo de medicamentos, material sanitario, pruebas adicionales de 


\section{Falla en la cicatrización post operatoria en apendicitis}

Vol. 3, núm. 3., (2019)

Nathalie María Barriga Reyes; Hypatia Amariles Ganan Macías; Renato Paolo Patiño Andrade; María Monserrate Ganchozo Pincay

diagnóstico y costos por día/cama; estableciéndose así, un significativo ahorro para el hospital. (Perión, 2017).

Lo señalado lleva a reconocer que, la apendicitis es catalogada como una de las enfermedades con mayor porcentaje de incidencia y causa de hospitalización en países latinoamericanos, esta alteración biológica se presenta de manera general, a través de un dolor agudo en el abdomen, a nivel de clínica es registrada como la mas frecuente emergencia quirúrgica abdominal y la cuarta operación más frecuente en la cirugía intra - abdominal. Por ello, su diagnóstico debe ser manejado, de manera profesional, bajo un estricto conocimiento de anatomía, fisiopatología y la clínica así como de las etapas que se producen en el desarrollo de esta enfermedad, pues, si bien, sus síntomas evolucionan en forma clásica, a veces hay cuadros atípicos que ponen al paciente en riesgo de padecer complicaciones.

A tal efecto, es de gran importancia que el diagnóstico de la apendicitis vaya acompañado de las pruebas normales de laboratorio, las mismas, apoyarán la definición del estudio diferencial, lo que es muy importante para dar un tratamiento adecuado, pues con el uso de estas pruebas se puede reducir la morbilidad y la mortalidad de los pacientes. Asimismo, estas enfermedades deben ser diagnosticadas de manera rápida y eficaz, mediante estudios clínicos actualizados, que brinden claridad a los indicios y características clínicas que presente cada paciente.

Como apoyo a lo anteriormente señalado, se resalta lo planteado en la Revista Chilena de Cirugía publicada por el Dr. (Perión, 2017), quien menciona que: 


\section{Falla en la cicatrización post operatoria en apendicitis}

Vol. 3, núm. 3., (2019)

Nathalie María Barriga Reyes; Hypatia Amariles Ganan Macías; Renato Paolo Patiño Andrade; María Monserrate Ganchozo Pincay

Antes de las 36 horas de iniciados los síntomas, el riesgo de rotura de la apéndice es muy baja y de menos del 2\%; pero a partir de ese plazo y cada 12 horas el riesgo aumenta significativamente, siendo mayor al 6\% cuando los síntomas sobrepasan las 36 horas. En tal sentido, es de gran importancia considerar que el diagnóstico a tiempo representa uno de los procesos de la medicina clínica que debe realizarse; su desarrollo minimizará los riesgos a que el paciente sufra complicaciones y pueda hasta perder la vida a causa de esta enfermedad. (p.23).

Por otra parte, se resalta la reseñado por (Mesa, 2016), en la Revista Mexicana "Cirujano General Núm. 2, quien menciona que, la medicación previa con analgésicos y antibióticos en pacientes con apendicitis aguda se asocia al retraso en la toma de la decisión quirúrgica; situación ésta que se encuentra asociada con el incremento de las complicaciones, como lo es, la perforación apendicular, el absceso de pared, el hemoperitoneo, el íleo postoperatorio, las colecciones abdominales, la evisceración, el hematoma de pared y óbitos .

En el mismo orden de ideas, se agrega que existe un alto porcentaje de pacientes que se retrasan 72 o más horas desde el inicio de los síntomas hasta el acto operatorio. Estos factores de demora, se ven condicionados por el hecho entre muchas causas, de que los pacientes no recurren a tiempo a los servicios de salud, lo que establece que estas personas, tengan un retraso considerable, deben ser intervenidos a tiempo, lo que los lleva a formar parte de un 2,5\% de la población en situación de riesgo clínico a padecer complicaciones médicas. 


\section{Falla en la cicatrización post operatoria en apendicitis}

Vol. 3, núm. 3., (2019)

Nathalie María Barriga Reyes; Hypatia Amariles Ganan Macías; Renato Paolo Patiño Andrade; María Monserrate Ganchozo Pincay

De lo antes expuesto, se evidencia que los Centros Hospitalarios de países latinoamericanos mantengan en la actualidad un alarmante porcentaje de pacientes con complicaciones en apendicitis aguda; específicamente aquellas de orden post operatorio, relacionadas con fallas de cicatrización, las cuales se encuentran asociadas a infecciones, lo que en consecuencia ocasiona un alto índice de afecciones, lo que se debe a que la demora permite que la enfermedad siga su evolución natural. Tal señalamiento, se encuentra apoyado por (Terranova, 2014), quien expone:

La apendicitis es un proceso evolutivo, secuencial, de allí las diversas manifestaciones clínicas y anatomo -patológicas que suele encontrar el cirujano y que dependerán fundamentalmente del momento o fase de la enfermedad en que es abordado el paciente, de allí que se consideren los siguientes estadíos: apendicitis congestiva o catarral, apendicitis flemonosa o supurativa, apendicitis gangrenosa o necrótica, apendicitis perforada. (p.24).

De aquí, que es importante considerar que, las complicaciones generadas en los post operatorios de apendicetomía dependen en gran porcentaje del momento o fase de esta enfermedad en que haya sido abordado clínicamente el paciente, quien de no recibir la asistencia médica adecuada pudiera presentar múltiples complicaciones, las cuales, al ser diagnosticadas, reflejan, de manera general la presencia de absceso residual e infección de herida post operatoria.

En efecto, los señalamientos antes descritos permiten revelar la presencia de una problemática en salud, demostrada en el gran número de pacientes que asisten a los Centros 


\section{Falla en la cicatrización post operatoria en apendicitis}

Vol. 3, núm. 3., (2019)

Nathalie María Barriga Reyes; Hypatia Amariles Ganan Macías; Renato Paolo Patiño Andrade; María Monserrate Ganchozo Pincay

Asistenciales de Salud de Latinoamérica presentando complicaciones médicas como lo es la falla en la cicatrización post operatoria y otras patologías asociadas; situación ésta que debe ser diagnosticada y atendida oportunamente, ya que de persistir en el tiempo pudieran originar el aumento de la morbi - mortalidad originada por las mismas, y en consecuencia el aumento de gastos de recursos médicos utilizados para este tipo de afecciones.

\section{Fuentes Documentales}

El análisis correspondiente a las características que predomina en el tema seleccionado, llevan a incluir diferentes fuentes documentales encargadas de darle el respectivo apoyo y en ese sentido cumplir con la valoración de los hechos a fin de generar nuevos criterios que sirven de referencia a otros procesos investigativos. Para (Arias, 2010), las fuentes documentales incorporadas en la investigación documental o bibliográfica, "representa la suma de materiales sistemáticos que son revisados en forma rigurosa y profunda para llegar a un análisis del fenómeno". (p.41). Por lo tanto, se procedió a cumplir con la realización de una lectura previa determinada por encontrar aquellos aspectos estrechamente vinculados con la "Falla en la cicatrización Post Operatoria en Apendicitis”, para luego explicar mediante un desarrollo las respectivas apreciaciones generales de importancia.

\section{Técnicas para la Recolección de la Información}

La conducción de la investigación para ser realizada en función a las particularidades que determinan a los estudios documentales, tiene como fin el desarrollo de un conjunto de acciones encargadas de llevar a la selección de técnicas estrechamente vinculadas con las características 


\section{Falla en la cicatrización post operatoria en apendicitis}

Vol. 3, núm. 3., (2019)

Nathalie María Barriga Reyes; Hypatia Amariles Ganan Macías; Renato Paolo Patiño Andrade; María Monserrate Ganchozo Pincay

del estudio. En tal sentido, (Arias Ob cit) refiere, que es "una técnica particular para aportar ayuda a los procedimientos de selección de las ideas primarias y secundarias”. (p. 71).

Por ello, se procedió a la utilización del subrayado, resúmenes, fichaje, como parte básica para la revisión y selección de los documentos que presentan el contenido teórico. Es decir, que mediante su aplicación de estas técnicas se pudo llegar a recoger informaciones en cuanto a la revisión bibliográfica de los diversos elementos encargados de orientar el proceso de investigación. Tal como lo expresa, (Arias Ob cit) "las técnicas documentales proporcionan las herramientas esenciales y determinantes para responder a los objetivos formulados y llegar a resultados efectivos" (p. 58). Es decir, para responder con eficiencia a las necesidades investigativas, se introdujeron como técnica de recolección el método inductivo, que hizo posible llevar a cabo una valoración de los hechos de forma particular para llegar a la explicación desde una visión general.

Asimismo, se emplearon las técnicas de análisis de información para la realización de la investigación que fue ejecutada bajo la dinámica de aplicar diversos elementos encargados de determinar el camino a recorrer por el estudio, según, (Arias, Ob cit) las técnicas de procesamiento de datos en los estudios documentales "son las encargadas de ofrecer al investigador la visión o pasos que debe cumplir durante su ejercicio, cada una de ellas debe estar en correspondencia con el nivel a emplear" (p. 123). Esto indica, que para llevar a cabo el procesamiento de los datos obtenidos, es necesario establecer las técnicas que serán seleccionadas, destacándose en este caso, de manera particular: fichas de resumen, textual, registros descriptivos entre otros, los mismos se deben ajustar al nivel que ha sido seleccionado. 


\section{Falla en la cicatrización post operatoria en apendicitis}

Vol. 3, núm. 3., (2019)

Nathalie María Barriga Reyes; Hypatia Amariles Ganan Macías; Renato Paolo Patiño Andrade; María Monserrate Ganchozo Pincay

\section{Resultados.}

\section{Apendicitis Aguda}

Es la inflamación del apéndice vermicular, seguida de su infección. Su tratamiento es quirúrgico y no electivo. Es la cirugía no electiva más común. Actualmente, se diagnostican y operan más de 250000 casos en Estados Unidos y 30000 casos por año en el Perú. Es muy frecuente en los hombres. Se da sobre todo en los adultos, entre los 20 y 30 años. El $1 \%$ de los afectados mueren, pero la mortalidad entre niños es más alta: 4 al 8\%. (Cent, 2016). El diagnóstico adecuado de esta alteración depende mucho de una buena historia clínica, un buen examen físico y una buena interpretación de los exámenes auxiliares. Esta alteración de la salud se resuelve sobre todo en forma quirúrgica.

\section{Cuadro clínico}

A pesar de los adelantos en los métodos de diagnóstico, la apendicitis aguda se diagnostica sobre todo clínicamente. La base para un buen estudio, depende de una historia clínica adecuadamente llenada, en la que se describa la forma de presentación del dolor, los síntomas asociados y los hallazgos del examen físico. La principal sintomatología en casi todos los pacientes es el dolor. Este aumenta gradualmente en las 24 horas siguientes al inicio. Este inicio es agudo y poco localizado. Su ubicación es epigástrica o peri umbilical, sin desaparecer con el decúbito. Este dolor se mueve a la fosa iliaca derecha, en el punto que se denomina de Mc Burney, aumentando su intensidad. Este fenómeno se da en el 50\% de casos. 


\section{Falla en la cicatrización post operatoria en apendicitis}

Vol. 3, núm. 3., (2019)

Nathalie María Barriga Reyes; Hypatia Amariles Ganan Macías; Renato Paolo Patiño Andrade; María Monserrate Ganchozo Pincay

\section{Figura $N^{\circ}$ 1. Apendicitis Aguda}

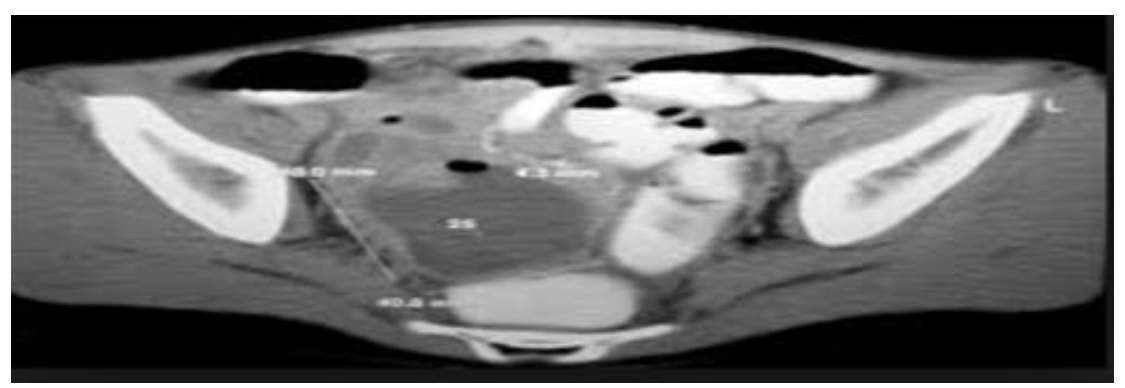

Fuente: Blanco (2011)

La presencia de dolor depende de la ubicación del apéndice. Si este se encuentra retro cecalmente, el dolor comenzara en alguna parte del hemi abdomen derecho. Si el apéndice es largo, yendo más allá de la línea media, podría haber dolor en el cuadrante inferior izquierdo. Por lo general habrá anorexia y nausea así como vómitos. Rara vez hay vómitos antes del dolor. La presencia de apetito hará dudar del diagnóstico. Existe una cronología en los síntomas: dolor, anorexia, incremento de la sensibilidad, fiebre y aumentos de leucocitos en sangre. Una alteración de esta cronología permitirá dudar del diagnóstico.

Los exámenes en el laboratorio son importantes para el diagnóstico diferencial. Por lo general se pide un hemograma y un examen de orina. La leucocitosis entre los 12000 y 18000 mm3 con predominio de neutrófilos sirve para confirmar el diagnóstico. Se cree que valores superiores a 18000 sugerirían la presencia de apendicitis con perforación. El examen urinario sirve para descartar la posibilidad de infección urinaria. Hasta el 33\% de los pacientes presentaran piuria y hematuria sin bacteriuria debido a que tanto el uréter y la vejiga están cerca del apéndice. 


\section{Falla en la cicatrización post operatoria en apendicitis}

Vol. 3, núm. 3., (2019)

Nathalie María Barriga Reyes; Hypatia Amariles Ganan Macías; Renato Paolo Patiño Andrade; María Monserrate Ganchozo Pincay

Por lo general la temperatura del cuerpo estará entre 37.5 y $38^{\circ} \mathrm{C}$. La fiebre no sirve para detectar la apendicitis, pero si hay taquicardia y fiebre puede haber perforación intestinal o un absceso intra abdominal. En estos casos durante la palpación abdominal existirá un resultado positivo durante la prueba del rebote, se encontrará resistencia abdominal voluntaria e involuntaria. Algunas veces se podrá palpar un plastrón.

\section{Complicaciones}

Se dan sobre todo cuando hay apendicitis perforada, con plastrón, con absceso y/o peritonitis. Las complicaciones más frecuentes son la peritonitis y los abscesos intra abdominales. Por lo general son infecciones poli microbianas debidas a la flora gastrointestinal. Los principales microorganismos son el Streptococcus spp, Escherichia Koli y K. Pneumoniae, los Bacteroides como fragilis, y vulgatus, B. distasonis, B. ovatus y B. thetaiotaomicron.

\section{Complicaciones sépticas}

\section{A) Parietales}

- Absceso superficial: Se dan entre el 4to y 7mo día luego de la operación. Habrá hipertermia sin infección, con regularidad en el tránsito intestinal. El diagnóstico se confirma a través del examen físico y la evaluación de la herida. El tratamiento será mediante la evacuación del absceso y el cierre de la herida quirúrgica por segunda intención.

- Gangrena parietal: No es frecuente, es muy rara. 


\section{Falla en la cicatrización post operatoria en apendicitis}

Vol. 3, núm. 3., (2019)

Nathalie María Barriga Reyes; Hypatia Amariles Ganan Macías; Renato Paolo Patiño Andrade; María Monserrate Ganchozo Pincay

\section{B) Peritoneales}

\section{- Peritonitis secundarias: Severa:}

- Fiebre.

- Regularización del tránsito intestinal parcial o presencia de diarreas.

- Desgaste o deterioro del estado general del paciente.

- Signos de infección.

- La causa puede ser por dehiscencia o necrosis de la ligadura del muñón o necrosis del fondo de saco. Es una emergencia. Es necesaria una nueva cirugía para retirar el absceso, lavar y drenar la cavidad.

- Abscesos intra - abdominales residuales: Se da entre el sexto y décimo día. Hay síntomas generales de infección, una restauración lenta del tránsito, diarrea con fiebre en picos. Es necesaria una ecografía. Se vuelve a intervenir cuando falla la técnica percutánea o hay contraindicaciones.

- Oclusiones: Según el tiempo que paso desde la cirugía se clasifican en:

- Precoces: Se dan en los 30 días posteriores a la operación. Su causa es adherencias.

- Tardías: Se da después de los 30 días. Su causa son las bridas. Se vuelve a hacer la cirugía para cortar las bridas. 


\section{Falla en la cicatrización post operatoria en apendicitis}

Vol. 3, núm. 3., (2019)

Nathalie María Barriga Reyes; Hypatia Amariles Ganan Macías; Renato Paolo Patiño Andrade; María Monserrate Ganchozo Pincay

\section{Factores de Riesgo}

Para (Blanco, 2015), un factor de riesgo representa una medida que refleja la probabilidad de que se produzca un hecho o daño a la salud (enfermedad o muerte). En todo caso, se puede entender que el mismo es "una característica o circunstancia detectable en un individuo o en grupos asociados con una probabilidad incrementada de experimentar un daño a la salud". (p.19). Es por ello que, el término factor de riesgo se usa con tres atributos distintos:

- Un atributo o exposición que se asocia con una probabilidad mayor de desarrollar un resultado específico tal como la ocurrencia de una enfermedad. Este atributo no necesariamente constituye un factor causal. (en este caso en especial, se refiere al diagnóstico (efectivo) y asistencia tardía del paciente a los Centros Hospitalarios), lo que en consecuencia aumenta la morbi mortalidad de cuadros médicos generados por apendicitis.

- Un atributo o exposición que aumenta la probabilidad de la ocurrencia de una enfermedad $\mathrm{u}$ otro resultado específico. (en este caso se encuentran los factores biológicos relacionados con patologías asociadas o, por ejemplo la automedicación del paciente ante el cuadro de dolor abdominal generado por la apendicitis)

- Un determinante que puede ser modificado por alguna forma de intervención logrando disminuir la probabilidad de la ocurrencia de alguna enfermedad o de otro daño específico a la salud, para evitar confusión esta connotación o parentesco debe ser referida como factor de riesgo modificable. 


\section{Falla en la cicatrización post operatoria en apendicitis}

Vol. 3, núm. 3., (2019)

Nathalie María Barriga Reyes; Hypatia Amariles Ganan Macías; Renato Paolo Patiño Andrade; María Monserrate Ganchozo Pincay

\section{Complicaciones por Falla de Cicatrización}

Las peritonitis y los abscesos intra abdominales son, por lo general, infecciones poli microbianas producidas por la flora saprofita del tracto gastrointestinal. Si bien los patógenos principales son Streptococcus spp. ,las enterobacterias (principalmente E. coli y K. pneumoniae) y los Bacteroides del grupo fragilis (B. fragilis, en sentido estricto y B. vulgatus, B. distasonis , B. ovatus y B. thetaiotaomicron), otros patógenos han ido ganando terreno aunque tradicionalmente hayan sido considerados como menores. Entre estos destacan E. faecalis $P$. aeruginosa y $C$. albicans.

\section{Imagen $N^{\circ}$ 2. Post Operatorio de Apendicitis}

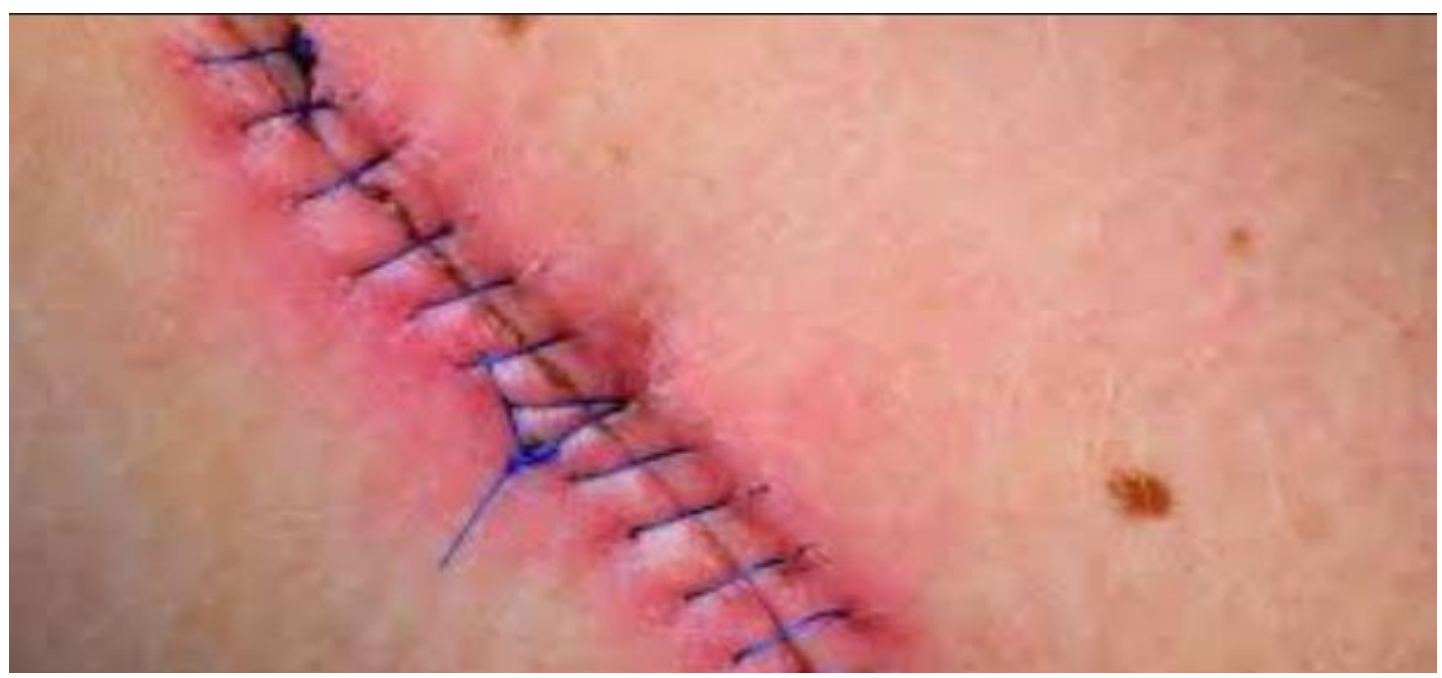

Fuente: Organización Mundial de la Salud (OMS 2017)

El hecho de que estos gérmenes se hayan considerado como secundarios se debe fundamentalmente a que no se aíslan tan frecuentemente como los patógenos más comunes y/o a su virulencia relativamente menor. Su tasa de aislamiento en las peritonitis adquiridas en la 


\section{Falla en la cicatrización post operatoria en apendicitis}

Vol. 3, núm. 3., (2019)

Nathalie María Barriga Reyes; Hypatia Amariles Ganan Macías; Renato Paolo Patiño Andrade; María Monserrate Ganchozo Pincay

comunidad se sitúa alrededor del 5-10\% y, por tanto, sólo de forma esporádica son causa de infección post operatoria intra abdominal o de herida en este contexto.

Por ende, las infecciones de heridas, por fallas en la cicatrización incluyen las que ocurren arriba de la fascia (infección superficial de la herida) y las que se presentan debajo de la misma (infección profunda de la herida). Algunos autores proponen los términos más inclusivos de infección del campo o del sitio quirúrgico, que comprenderían todos los sitios quirúrgicos expuestos potencialmente a bacterias. Estos términos abarcarían infecciones superficiales y profundas de heridas y las que no ocurren en proximidad directa con la incisión quirúrgica (ej. Absceso intra abdominal postoperatorio).

Los criterios de Infección del Sitio Operatorio (antes denominada Infección de Herida Quirúrgica u Operatoria) han variado ampliamente a lo largo del tiempo. Según la definición clásica de Ljungquist se consideraba una herida quirúrgica infectada aquella que desarrollaba una colección de pus. En 1980, en el proyecto SENIC (Study on the Efficacy of Nosocomial Infection Control) definió la infección o falla en la cicatrización de sitio operatorio en base a los criterios de los de la CDC (Centers for Disease Control and Prevention) para el National Nosocomial Infeccions Surveillance System (NNISS), tal y como se describe:

\section{Infección Incisional del Sitio Operatorio: Diagnóstico por cualquiera de los siguientes} criterios:

[1] Diagnóstico hecho por el médico.

[2] Drenaje purulento de la herida.

[3] Fiebre y eritema, o separación de los bordes, o cultivo positivo, sin drenaje de pus. 


\section{Falla en la cicatrización post operatoria en apendicitis}

Vol. 3, núm. 3., (2019)

Nathalie María Barriga Reyes; Hypatia Amariles Ganan Macías; Renato Paolo Patiño Andrade; María Monserrate Ganchozo Pincay

\section{Infección Profunda del Sitio Operatorio: Diagnóstico por cualquiera de los siguientes criterios:}

[1] Diagnóstico hecho por el médico.

[2] Drenaje purulento por un tubo de drenaje, fístula o abertura natural del cuerpo.

[3] Encontrada al re operar la zona.

\section{Imagen $N^{\circ}$ 3. Diagnóstico de Apendicitis Aguda}

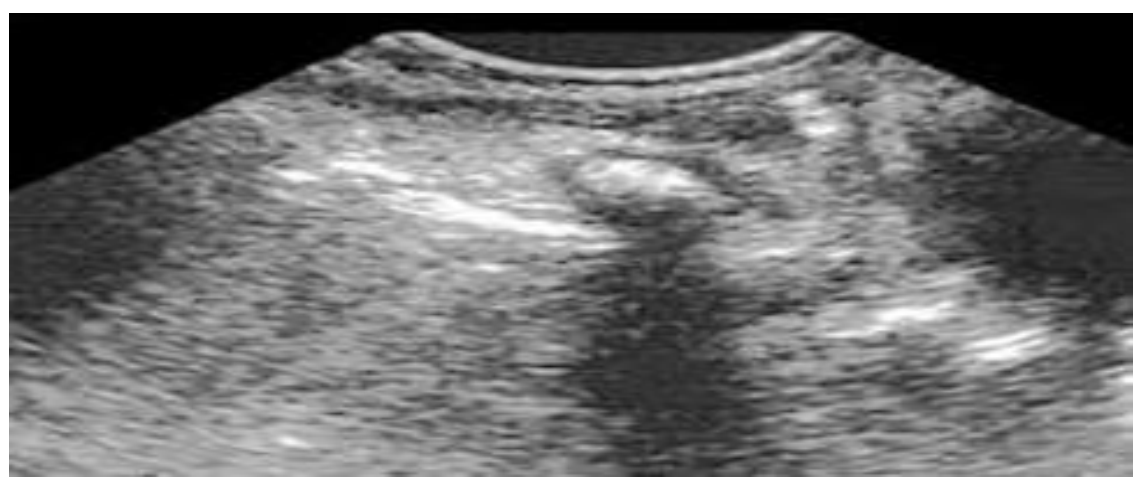

Fuente: Organización Mundial de la Salud (OMS 2017)

Los conocimientos actuales de la cadena epidemiológica de las infecciones y principalmente de sus mecanismos de transmisión, nos indican la necesidad de implantar en todo el ámbito asistencial (tanto intra como extra hospitalario), prácticas de asepsia y antisepsia imprescindibles para el control y la prevención de las infecciones. En este sentido, el objetivo final del servicio de esterilización y suministro es la distribución del material hospitalario estéril o desinfectado, contribuyendo desde ya al proceso general de asepsia y antisepsia en los diferentes procedimientos que se realicen en la institución. 


\section{Falla en la cicatrización post operatoria en apendicitis}

Vol. 3, núm. 3., (2019)

Nathalie María Barriga Reyes; Hypatia Amariles Ganan Macías; Renato Paolo Patiño Andrade; María Monserrate Ganchozo Pincay

En 1839, Justin von Liebig (químico) sostuvo que la sepsis era una especie de combustión causada por la exposición de los tejidos húmedos al oxígeno, y por esta razón se consideraba que la mejor forma de prevenirla era evitando que el aire entrara a la herida. Joseph Lister había observado esas heridas infectadas y consideraba que la sepsis de las heridas era más bien una especie de descomposición. En 1865, Louis Pasteur sugirió que la descomposición era causada por microorganismos en el aire que al ponerse en contacto con la materia la fermentaban.

Por esta razón los microorganismos debían ser destruidos antes de que entraran a la herida. Los agentes pioneros de los antisépticos generalmente no fueron aceptados en las publicaciones de Pasteur, sino hasta 1863, cuando se reconoció el origen microbial de la putrefacción. Sommelweis, en 1847, introdujo la práctica del lavado de las manos con compuestos clorinados. Joseph Lister (1827-1912), años después, amplió el uso a soluciones fenólicas, tanto para el lavado de las manos como para el lavado de la piel de los pacientes, de la ropa y del instrumental usado.

Una solución al 2,5\% fue usada para vendaje de heridas y a doble concentración para esterilizar instrumentos. Estos conceptos basados inicialmente en la observación y posteriormente en los conceptos microbiológicos, los cuales lograron un impacto importante en la prevención de las infecciones intrahospitalarias, y abrió el camino para el gran avance en la cirugía. John Pringle parece ser el primero en usar el término antiséptico en 1750 para describir sustancias que previenen la putrefacción. La idea fue eventualmente aplicada para el tratamiento de las heridas supuradas. 


\section{Falla en la cicatrización post operatoria en apendicitis}

Vol. 3, núm. 3., (2019)

Nathalie María Barriga Reyes; Hypatia Amariles Ganan Macías; Renato Paolo Patiño Andrade; María Monserrate Ganchozo Pincay

Alrededor de 1870, el uso de los antisépticos todavía era empírico, no habiendo mayor desarrollo. Muchos antisépticos tradicionales se han continuado usando en forma más refinada, los fenoles se han modificado y se han hecho más aceptables para uso general. La acriflavina, introducida en 1913, fue el primer miembro de los antisépticos básicos, estuvo muchos años en uso, pero fue desplazada en las tres últimas décadas por los antisépticos catiónicos incoloros. No obstante, a pesar del amplio uso en la actualidad de los antimicrobianos, no se ha eliminado el uso de los antisépticos y desinfectantes, al contrario se ha perfeccionado las fórmulas de aquellas sustancias químicas como el yodo y otros más recientes como la clorhexidina.

En razón de lo anteriormente mencionado, se establece que, el riesgo de infección o falla de la cicatrización luego de un operatorio es muy variable y depende del procedimiento quirúrgico y de la presencia de factores de riesgo. Como tales se deben considerar a aquellas variables que tienen una relación independiente y significativa con el desarrollo de una infección del sitio operatorio. El conocimiento de dichos factores de riesgo permite estratificar adecuadamente las diferentes intervenciones realizadas y así, controlar las infecciones de una forma más racional.

También, facilita a la adopción de medidas preventivas que irán dirigidas a disminuir la posibilidad de contaminación de la herida (medidas de asepsia y antisepsia), a mejorar el estado general o local del paciente o a evitar la transformación de la contaminación en infección (profilaxis antibiótica). Por ende, el riesgo de infección de una herida depende en parte de la posibilidad de contaminarse durante el acto operatorio. Para estimar este riesgo, el Consejo de Investigación de la Academia Nacional de Ciencias de los EE.UU propuso en 1964 un modelo 


\section{Falla en la cicatrización post operatoria en apendicitis}

Vol. 3, núm. 3., (2019)

Nathalie María Barriga Reyes; Hypatia Amariles Ganan Macías; Renato Paolo Patiño Andrade; María Monserrate Ganchozo Pincay

para clasificar los diferentes tipos de herida quirúrgica; este sistema, con algunas modificaciones, es el que se utiliza actualmente e involucra en su identificación las siguientes causas:

Herida Limpia: Herida realizada durante una cirugía electiva con cierre primario y en ausencia de todos los siguientes:

- Colocación de drenajes por la herida

- Violación de técnica aséptica

- Evidencias de infección

- Apertura de mucosas.

Herida Limpia- Contaminada: Herida quirúrgica con al menos una de las siguientes condiciones:

- Apertura de mucosas sin evidencias de infección

- Derrame mínimo del contenido intestinal en la cavidad

- Violación mínima de la técnica aséptica

- Colocación de drenajes por la herida.

Herida Contaminada: Herida quirúrgica o traumática con al menos una de las siguientes condiciones:

- Apertura de mucosas con evidencia de infección y sin pus

- Derrame grosero del contenido intestinal en la cavidad 


\section{Falla en la cicatrización post operatoria en apendicitis}

Vol. 3, núm. 3., (2019)

Nathalie María Barriga Reyes; Hypatia Amariles Ganan Macías; Renato Paolo Patiño Andrade; María Monserrate Ganchozo Pincay

- Violación mayor de la técnica aséptica

- Herida traumática dentro de las 4 horas de producido el accidente.

Herida Sucia: Herida quirúrgica o traumática con al menos una de las siguientes condiciones:

- Apertura de tejidos con evidencias de inflamación purulenta

- Herida traumática luego de las 4 horas de producido el accidente

- Herida contaminada con materia fecal o con cualquier otro material infectante.

Las infecciones postoperatorias de las heridas se originan de la contaminación bacteriana durante o después de una operación.

Bacterias de la Flora Habitual: La clasificación de la herida quirúrgica según su grado de contaminación está basada precisamente en el tipo de flora que se encuentra al abrir las diferentes cavidades u órganos durante la cirugía, ya que es a partir de ellos y en el momento de la cirugía cuando se van a adquirir la mayor parte de las infecciones de sitio operatorio.

Microflora cutánea: La flora cutánea comprende bacterias comensales (o residentes), transeúntes así como potencialmente patógenas. Las comensales incluyen bacterias aerobias y anaerobias tales como Propionibacterias (difteroides y coryneformes) y Staphylococcus epidermidis. Este último organismo coagulasa negativo es un patógeno potencial en la cirugía protésica donde particularmente la adquisición nosocomial de formas multi resistentes es un hecho frecuentemente informado. Los organismos transeúntes, que no se encuentran normalmente en la piel, incluyen Staphilococcus aureus (meticilín resistente o no) y coliformes, los cuales poseen un elevado potencial infeccioso. 


\section{Falla en la cicatrización post operatoria en apendicitis}

Vol. 3, núm. 3., (2019)

Nathalie María Barriga Reyes; Hypatia Amariles Ganan Macías; Renato Paolo Patiño Andrade; María Monserrate Ganchozo Pincay

Otros patógenos que pueden contaminar temporalmente la piel incluyen Streptoccus pyogenes, Bacteroides, Clostridia (como esporas) y Candida. Estos microrganismos están particularmente asociados con la contaminación de la piel ocasionada por el afeitado o por una pobre higiene preoperatoria. La población bacteriana de la piel normal en pacientes sanos se controla por la sequedad de la piel, el pH ácido así como por la descamación y por el elevado contenido en sales. Los lípidos de la piel poseen propiedades antimicrobianas, como las secreciones de otros epitelios especializados que también aclaran bacterias a través de los macrófagos y por la acción ciliar.

Microflora intestinal: El tubo digestivo es una enorme superficie de interacción del individuo con el medio externo, que alberga en su conjunto un número de células microbianas que excede en mucho el número de células del organismo humano. El intestino es extremadamente variable en sus condiciones ecológicas, de forma que las interacciones microorganismos-hábitat podrían considerarse en cada uno de sus tramos como correspondientes a ecosistemas diferentes.

Así, el estómago, debido a su pH ácido ejerce una función de barrera a la colonización por la mayoría de los microorganismos, no existiendo más de 103 microorganismos por gramo de pared de estómago que corresponde habitualmente a flora oral y oro faríngea deglutida (Streptococcus,aphylococcus, Lactobacillus o Peptostreptococcus). Únicamente ciertas bacterias con sistemas de protección especial como Helicobacter podrían considerarse como flora residente del estómago. 


\section{Falla en la cicatrización post operatoria en apendicitis}

Vol. 3, núm. 3., (2019)

Nathalie María Barriga Reyes; Hypatia Amariles Ganan Macías; Renato Paolo Patiño Andrade; María Monserrate Ganchozo Pincay

El intestino delgado como área de mayor importancia en el proceso digestivo y absortivo tiende a controlar su carga microbiana, ya que los microorganismos podrían limitar el acceso de nutrientes a los enterocitos y competir con el huésped en su aprovechamiento. La cantidad de bacterias va aumentando a medida que se avanza en el intestino delgado, existiendo recuentos de 104-107 bacterias/ml en el íleon.

El intestino grueso constituye el mayor contenedor de microorganismos del cuerpo humano, ya que reúne las condiciones para ser colonizado (relativa deshidratación, baja peristalsis, pH próximo a la normalidad y gran eliminación de moco, con capacidad adhesiva y multiplicativa para las bacterias). La válvula íleocecal es la frontera que delimita el mayor ecosistema microbiano integrado en el hombre.

Factores Pronósticos: Diferentes estudios han identificado una serie de factores de riesgo que pueden influir en la aparición de la infección de sitio operatorio; entre otros cabe destacar:

\section{(1) Intrínsecos (relacionados con el paciente)}

- Edad avanzada.

- Enfermedades asociadas (diabetes, cáncer, enfermedad vascular crónica y obesidad).

- Alteración de la función inmune por enfermedad o regímenes terapéuticos.

- Tabaquismo.

- Desnutrición.

- Infección recurrente en un lugar remoto.

- Perfusión tisular disminuida.

(2) Factores Extrínsecos (relacionados con la cirugía y el ambiente hospitalario)

- Lavado de manos para el acto quirúrgico. 


\section{Falla en la cicatrización post operatoria en apendicitis}

Vol. 3, núm. 3., (2019)

Nathalie María Barriga Reyes; Hypatia Amariles Ganan Macías; Renato Paolo Patiño Andrade; María Monserrate Ganchozo Pincay

- Preoperatorio prolongado.

- Hospitalización prolongada.

- Operaciones anteriores.

- Vestuario quirúrgico.

- Duración de la cirugía. - Instrumental.

- Técnica quirúrgica.

- Antisepsia de la piel.

- Antibióticos profilácticos.

- Esterilización.

Antibióticos Profilácticos: La administración de antibióticos puede reducir la frecuencia de infección post operatorio de la herida en pacientes con operaciones electivas. Existen ciertos principios que rigen la profilaxis con antibióticos. Debe elegirse antibiótico terapia profiláctica contra las bacterias que se creen que contaminarán la herida. En operaciones limpias en la que es apropiada la profilaxis con antibióticos, los gérmenes que con mayor probabilidad originan infecciones son S. aureus, S. epidermidis y bacterias entéricas gram-negativas. Estas últimas son la causa más probable de infecciones de heridas después de 43 operaciones gastroduodenales y en vías biliares, cirugía colorrectal, apendicetomía y cirugía ginecológicas.

\section{Conclusiones.}

La apendicitis, representa un proceso inflamatorio bacteriano de la apéndice cecal. El factor causal predominante en el desarrollo de la patología es la obstrucción de la luz, la causa usual de ésta es la obstrucción por fecalitos, otros menos frecuentes son la hipertrofia del tejido linfoide e impacto de semillas y gusanos intestinales. La prolongación de las horas de evolución 


\section{Falla en la cicatrización post operatoria en apendicitis}

Vol. 3, núm. 3., (2019)

Nathalie María Barriga Reyes; Hypatia Amariles Ganan Macías; Renato Paolo Patiño Andrade; María Monserrate Ganchozo Pincay

en más de 25 horas tiene una relación con la presencia de heridas contaminadas y sucias, lo cual es evidente en los resultados quirúrgicos, y es un factor de riesgo que favorece la aparición de infección de sitio operatorio.

La ocurrencia de una infección o falla post operatoria en la herida requiere de la coexistencia de algunos factores; inoculación suficiente del germen y disminución en los mecanismos de defensa y alteraciones en las células. La profilaxis antibiótica utilizada para estos casos, es considerado como un factor menor para disminuir el riesgo de infección, pero su eficacia e impacto están claramente demostrados, así como su significancia.

Las infecciones de la herida causan un gran impacto en la calidad de vida del paciente y en los costos hospitalarios. Las consecuencias sobre el paciente van desde aumento del dolor hasta el manejo de una herida abierta, sepsis y hasta la muerte. El uso de antibióticos postoperatorios en los pacientes apendicectomizados con apéndices no complicados, no disminuye el riesgo de complicaciones infecciosas como lo son los abscesos de la herida operatoria y las colecciones intra-abdominales.

\section{Bibliografía.}

Arias, F. (2010). Paradigmas, Método y Enfoque de la Investigación Científica. España: Editorial: Luces.

Blanco, D. (2015). Factores de Riesgo de la Apendicitis Aguda. Revista Médica de la Universidad de los Andes ULA, Mérida, Venezuela, 12.

CDS. (2017). Apendicitis Aguda. Problema de Salud Pública. Revista Vida y Salud de la Universidad de la Habana en Cuba, 13.

Cent, S. (2016). Tratamiento de apendicitis aguda: Evaluación. Revista Salud de Buenos Aires, Argentina, 22. 


\section{Falla en la cicatrización post operatoria en apendicitis}

Vol. 3, núm. 3., (2019)

Nathalie María Barriga Reyes; Hypatia Amariles Ganan Macías; Renato Paolo Patiño Andrade; María Monserrate Ganchozo Pincay

Dávila, N. (2012). Paradigmas de la Investigación Científica. Pereire, Colombia: Editorial: Las Brisas.

Gumter, J. (2016). Factores de Riesgo que inciden en complicaciones de Apendicitis Aguda. Salud Integral de la Universidad Autónoma de México, 14.

Humt, B. (2017). Intrabdominal Absceso. Apendicitis y sus Complicaciones. American Jornal of Surgery, 27.

Mesa, A. (2016). Apendicitis Aguda y sus Complicaciones. Revista Méxicana "Cirujano General", 18.

Perión, C. (2017). Infecciones Gástricas Post Operatorias. Revsita Chilena de Cirugía, 14.

Salud, O. M. S. (2017). Epidemiología de la Apendicitis Aguda en países Latinoamericanos. Revista Mexicana "Cirujano General", 23.

Terranova, M. (2014). Apendicitis un proceso evolutivo Secuencial. Revista Salud Integral de la Universidad de la Rioja en Colombia, 23.

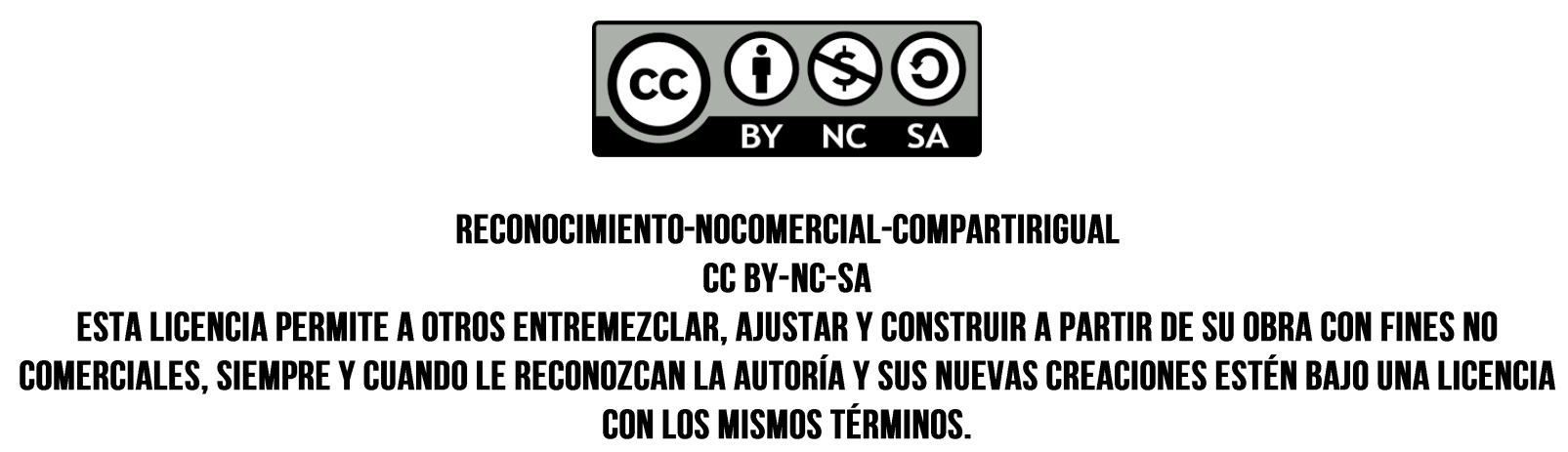

